\title{
The Five Years Surveillance and Trends of Antibiotic Resistance in Some Common Gram Negative Bacteria at the Vietnam Military Hospital
}

\author{
Ha Thi Thu Van1, Hoang Xuan Quang1, Vo Thi Bich Thuy², Nguyen Thai Son ${ }^{*}$ \\ ${ }^{1}$ Military Hospital 103, Vietnam Military Medical University, Hanoi, Vietnam \\ ${ }^{2}$ Institute of Genome Research, Vietnam Academy of Science and Technology, Hanoi, Vietnam \\ Email: *sonvsv@gmail.com
}

How to cite this paper: Van, H.T.T., Quang, H.X., Thuy, V.T.B. and Son, N.T. (2020) The Five Years Surveillance and Trends of Antibiotic Resistance in Some Common Gram Negative Bacteria at the Vietnam Military Hospital. Advances in Microbiology, 10, 730-741.

https://doi.org/10.4236/aim.2020.1012052

Received: November 26, 2020

Accepted: December 28, 2020

Published: December 31, 2020

Copyright $\odot 2020$ by author(s) and Scientific Research Publishing Inc. This work is licensed under the Creative Commons Attribution International License (CC BY 4.0).

http://creativecommons.org/licenses/by/4.0/

(c) (i) Open Access

\begin{abstract}
The study is to identify trends and levels of antibiotic resistance of some common Gram-negative strains over time. The samples were collected from Vietnam Military Hospital 103 between 2014 and 2019. A total of 405 Acinetobacter baumannii, 528 Pseudomonas aeruginosa, 741 Escherichia coli, and 352 Klebsiella pneumoniae strains were identified and antimicrobial susceptibility was by Vitek system and Etest method. The multi-drug resistance (MDR) was major proportion of four common bacteria. In particular, there is a tendency to shift from MDR to Extended drug resistance (XDR) or possibly Pan drug resistant (pPDR). A. baumannii had the highest level of antibiotic resistance, namely, carbapenem $(61.5 \%$ - 82.5\%) and cephalosporin $(72.7 \%$ $88.7 \%)$. $P$. aeruginosa resisted most of commonly antibiotics, ranging from $50 \%$ to $70 \%$. E. coli had a high resistance with antibiotics like ampicillin $(87.2 \%-97.6 \%)$ and the 3 rd generation cephalosporins (up to $79.6 \%) . K$. pneumoniae resisted carbapenem from $14.7 \%$ to $44.4 \%$, and other antibiotics with the higher rate of $40 \%$. The collected data will be a prerequisite for further studies on mechanisms and factors related to antibiotic resistant bacteria, in order to find out a rational and effective using strategy of antibiotics.
\end{abstract}

\section{Keywords}

Gram-Negative Bacteria, Antibiotic Resistance, Multi-Drug Resistance

\section{Introduction}

The invention of antibiotics is an immense achievement in the treatment of in- 
fectious diseases. However, the widespread use of antibiotics in the past decades has led to the emergence of multi-drug resistant bacteria. It caused a difficulty in infection treatment, influenced on the cost and days of treatment. It is a risk of facing global health sector [1].

Recognizing the importance of antibiotic-resistance of bacteria, global scientists and Vietnamese ones continuously research with a hope of reducing and controlling this problem. In February 2017, the World Health Organization (WHO) released a list of 12 strains of antibiotic-resistant bacteria that should be a top priority in researching and discovering new antibiotics [2]. Gram-negative bacterial strains are primary concern because of their potential to cause hospital infections and drug resistant level. Among the agents of Gram-negative bacteria, four common bacteria A. baumannii, P. aeruginosa, E. coli and K. pneumoniae are the most mentioned species studies. Because they are not only the causes of serious infections such as sepsis, pneumonia, urinary infections and hospital infections but also the agents that are resistant to many common antibiotics [3]. Unsurprisingly, most of the mentioned strains have appeared in Vietnam. The concern is that these strains have resisted many different antibiotics and increased resistant levels over time [4] [5].

Gram-negative bacteria have been always mentioned by many studies, because their antibiotic resistance has changed over time and geographic area [6]. It is necessary to regularly monitor and study the trends as well as the level of resistance of bacteria. The aim for the clinicians has a reasonable and effective strategy in use and store antibiotics in different areas in order to improve treatment quality and limit transmission of multi-resistant strains. In addition, the results also provided extra data to finalize the global report of antibiotic resistant bacteria.

In fact, there is a great difficulty in choosing antibiotics for treatment. So, this study focuses on showing kinds of antibiotics that the common bacteria are increasing their resistance and/or sensitive in recent years.

\section{Materials and Methods}

\subsection{Clinical Samples, Identification and Antimicrobial Susceptibility Tests of Gram-Negative Bacteria}

Clinical specimens were collected from patients at the military hospital in Vietnam during 2014-2019. The isolation techniques were conducted according to WHO standards and specialized microbiological guidance of Vietnam Ministry of Health [7] [8]. The species were identified using the Vitek 2 GN ID card. Antimicrobial susceptibility testing of isolates was performed on the Vitek 2 Antimicrobial Susceptibility Test (bioMérieux, France) [9], determined the minimum inhibitory concentration of antibiotics (MIC) by Etest (bioMérieux, France) method [10]. Results of antimicrobial susceptibility tested were interpreted according to Clinical and Laboratory Standards Institute guidelines, updated every year [11]. The quality of the identification procedure was controlled 
by using the Enterobacter homachchei ATCC 700324 strain. The strains of $P$. aeruginosa ATCC 27853 and E. coli ATCC 25922 were used to control the quality of antibiotic sensitivity tests.

\subsection{Statistical Analyses}

The data collected during the research period of the project is processed according to medical statistical methods, using WHONET 2019 antibiotic management and analysis software. Comparative testing using EpiCal 2000 software, the $\mathrm{p}$ values $<0.05$ was considered statistically significant.

\section{Results}

\subsection{Characteristics of Drug Resistant Trends}

For all four common Gram-negative bacteria, the rates of multi-drug bacteria were higher than those of resistant bacteria under 3 antibiotic groups $(\mathrm{p}<0.05)$. In particular, the rate of multi-resistant of $A$. baumannii tended to increase gradually over the years of research (Figure 1).

The multi-drug resistant (MDR) strains were major proportion of the 4 species of common bacteria. In particular, there is a tendency to shift from MDR to XDR (Extended drug resistance, non-susceptible in all but $\leq 2$ categories) or possibly PDR (Pan drug resistant, non-susceptible to all antimicrobial listed) in the studied bacteria. The resistant $A$. baumannii were almost XDR or pPDR. The rates of them fluctuated from $95 \%$ to $100 \%$ over the years. The trend of increasing XDR or PDR strains was also evident in P. aeruginosa at $67.86 \%$ in 2014 and gradually reached to $90.32 \%$ in 2019 ( $\mathrm{p}=0.02$ ) (Figure 2 ). We could not test with all of antibiotic groups, so they did not affirm which strains PDR was, and only called it pPDR.

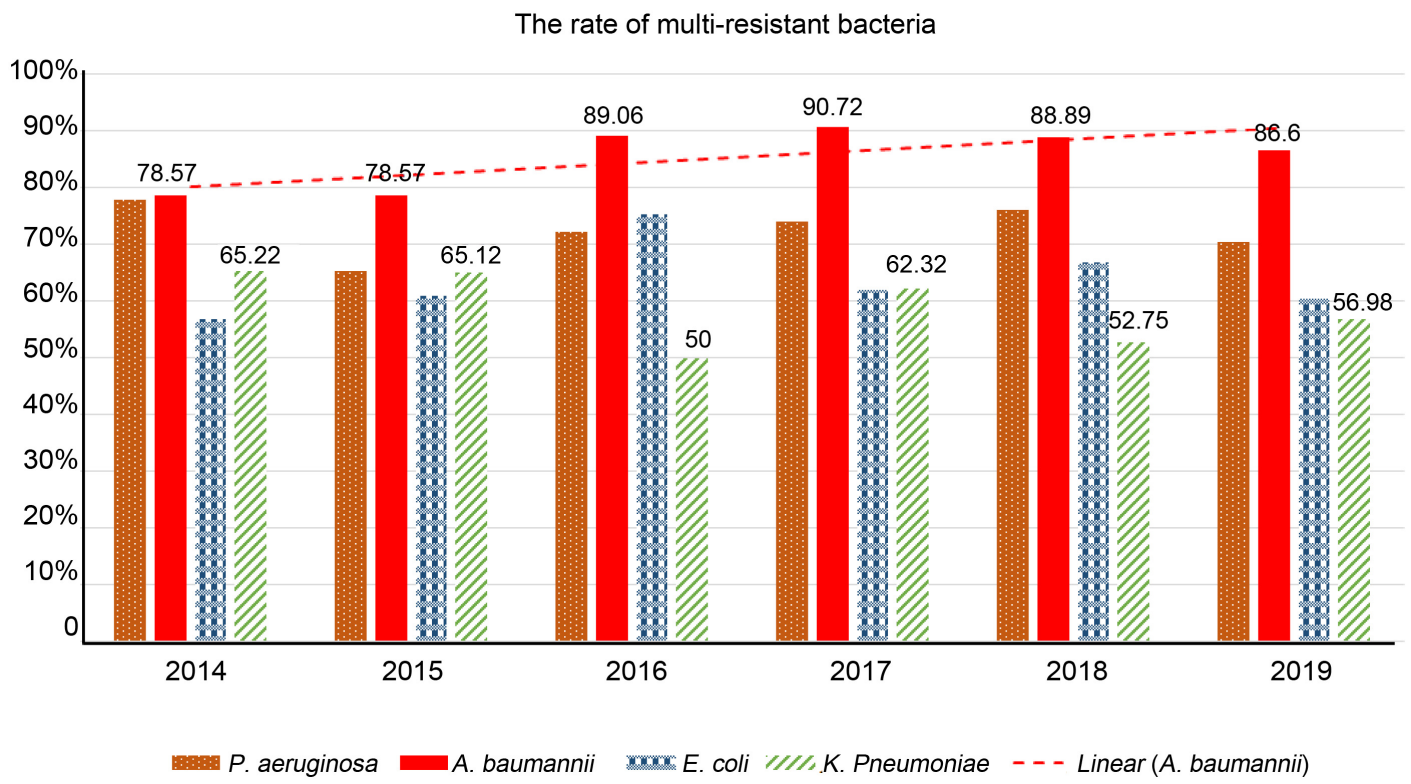

Figure 1. Distribution of multi-resistant bacteria over time. 


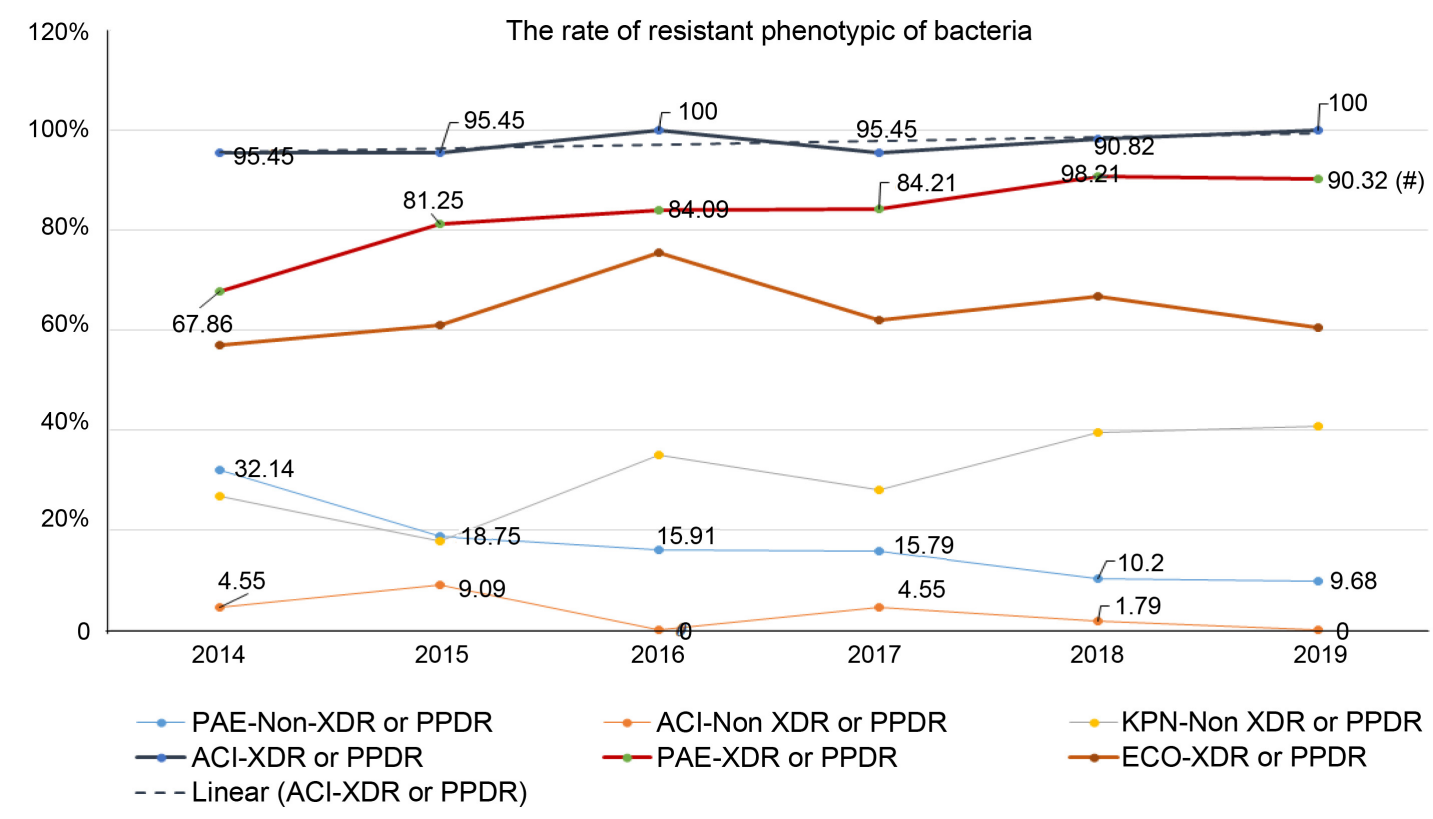

Figure 2. Distribution of MDR (Non - XDR or pPDR bacteria) and XDR or pPDR (possible PDR) bacteria on total multidrug - resistant bacteria over time. ${ }^{*} \mathrm{~A}$ statistically significant result $(\mathrm{p}=0.02)$ between XDR and PDR during 2014-2019.

\subsection{The Level of Antibiotic Resistance of Some Gram-Negative Bacteria Isolated}

A. baumannii was resistant with almost common antibiotics and tended to increase over time. Between 2017 and 2019, the antibiotic resistant rates of $A$. baumannii were over $80 \%$ (Figure 3 ).

The rate of resistance with imipenem and meropenem in 2014 was $61.5 \%$ and $70.8 \%$. In 2019, both of rates increased to $81.8 \%$. Amikacin and co-trimoxazole were still susceptible with $A$. baumannii. The level of resistance tended to decrease over time, but the overall resistant rates with these were quite high, at $30.0 \%$ and $37.4 \%$ respectively. Figure 4 indicated that the typical level of antibiotic resistance in $P$. aeruginosa was high $(>50 \%)$ and tended to increase over time $(\mathrm{p}<0.05)$. This bacterium was resistant with quinolones at a very high rate: levofloxacin $67.4 \%$ and ciprofloxacin $66.6 \%$. The carbapenem antibiotics such as imipenem and meropenem had resistant rates at $57.3 \%$ and $59.7 \%$, respectively. Resistance of $P$. aeruginosa with other antibiotics such as ceftazidime (52.0\%), cefepime $(48.7 \%)$, ticarcillin (68.2\%), azetreonam $(60.9 \%)$, and tobaramycin (58.4\%) was shown. Two antibiotics had effects on $P$. aeruginosa were amikacin $(20.9 \%-40.9 \%)$ and piperacillin $(15 \%-39.4 \%)$. However, the antibiotic resistant level of bacterium with these antibiotics was increasing over time, especially with a continuous increase from 2017 to 2019 (Figure 4).

$E$. coli had a high resistance to antibiotics, such as ampicillin $(>80 \%)$, cephalosporin group (up to 79.7\%), quinolone and flouroquinolone (range from $40.9 \%$ to $72.6 \%)$, co-trimoxazole $(75.8 \%)$. When considering the trend of changing resistant level by year, $E$. coli tended to increase resistance to $3^{\text {rd }}$ 


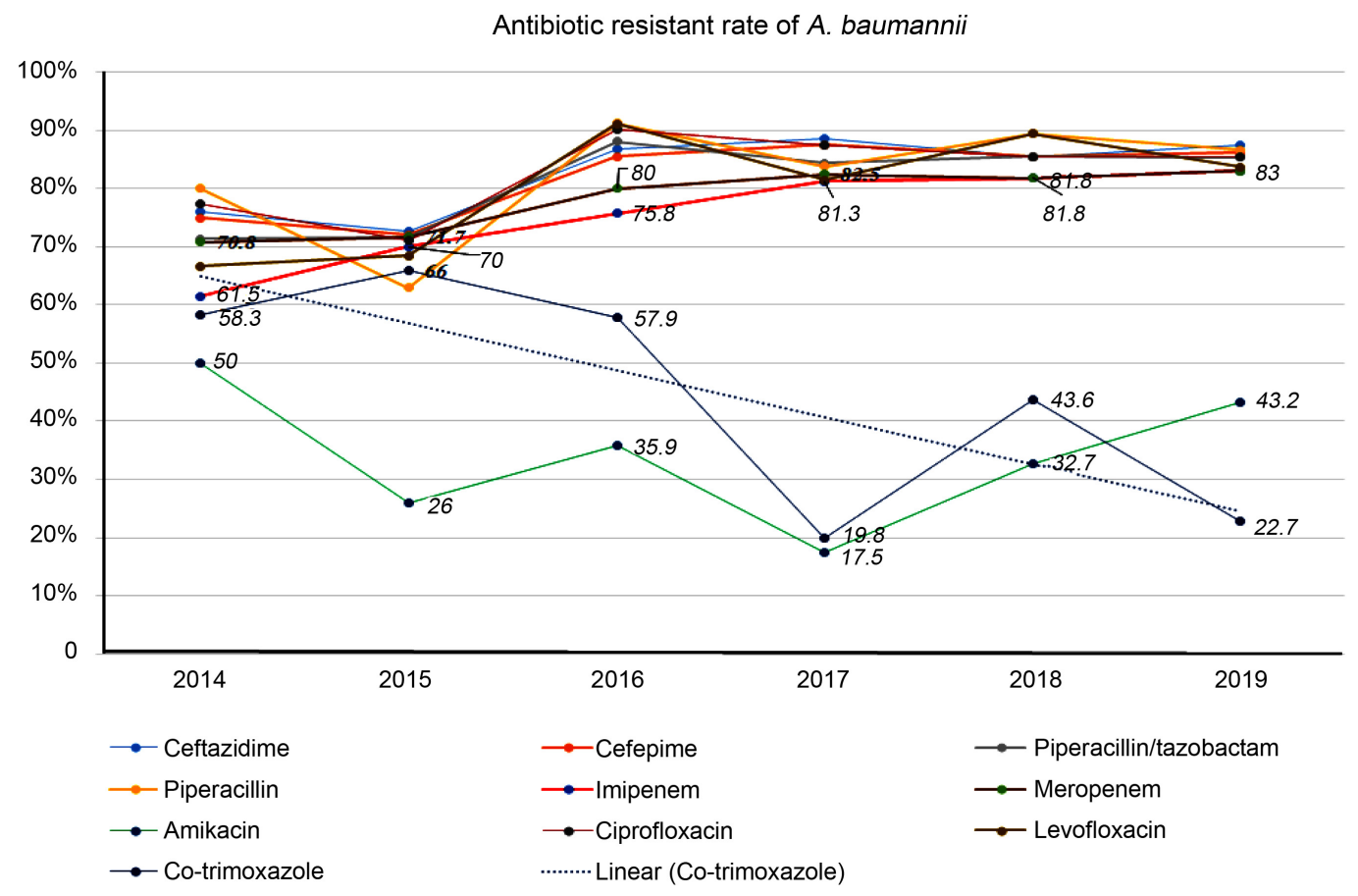

Figure 3. The resistant level of $A$. baumannii over time.

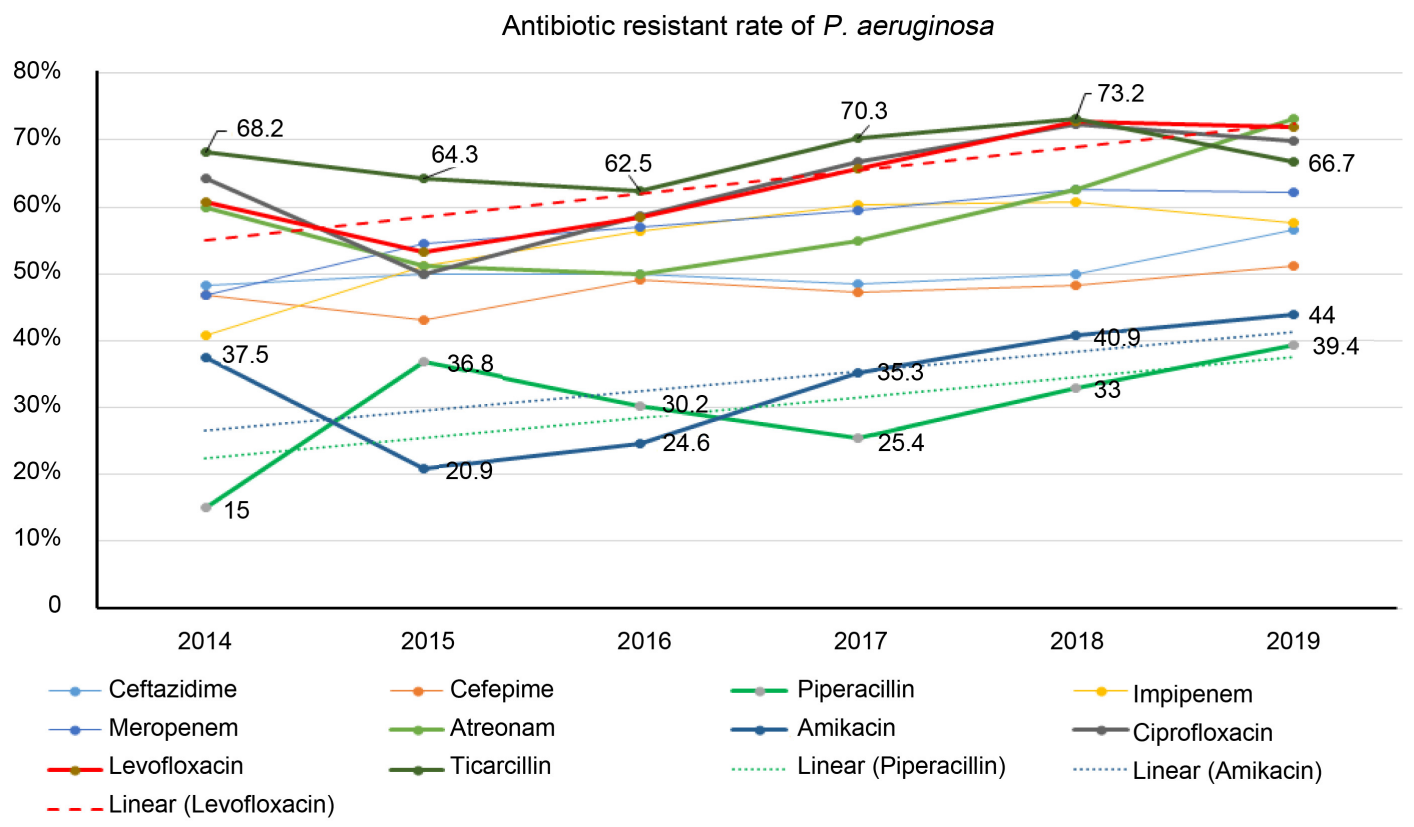

Figure 4. The resistant level of $P$. aeruginosa over time.

generation cephalosporins and quinolones (norfloxacin and ciprofloxacin) ( $\mathrm{p}<$ $0.05)$. However cefepim, there was a slight decrease in the resistant rates but the difference between annual rates was not significant $(\mathrm{p}>0.05)$ (Figure 5).

Klebsiella pneumoniae was resistant to many antibiotics such as cephalosporins, quinolones, aminoglycosides, and co-trimoxazole with the ratios from $25.8 \%$ to $60.7 \%$. The level of resistance to many antibiotics increased in 2015 and 
2017, especially the resistance to carbapenem antibiotics in 2017 was $44.6 \%$, approximately 2 times higher than the remaining years. The trends of increasing resistance to meropenem, imipenem and cefepim antibiotics were statistically significant $(\mathrm{p}<0.05)$. There was a notable reduction in resistance to ertapenem, fosfomycin, amikacin, amoxicillin/calvulanic acid (Figure 6).

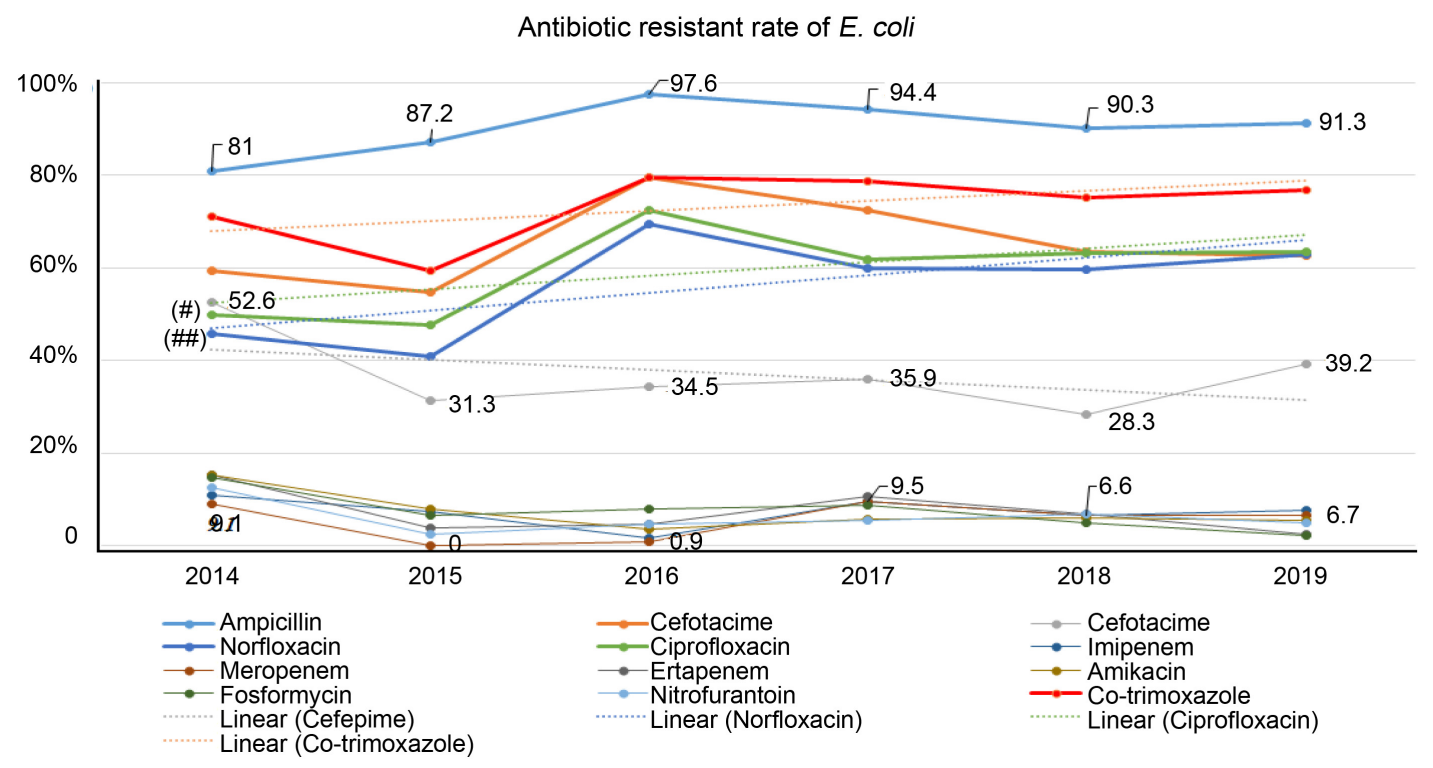

Figure 5. The resistant level of $E$. coli over time. ${ }^{*} \mathrm{p}<0.05$, a statistically significant result in Norfloxacin resistance during 2014-2019. ${ }^{\#} \mathrm{p}<0.05$, a statistically significant result in Ciprofloxacin resistance during 2014-2019.

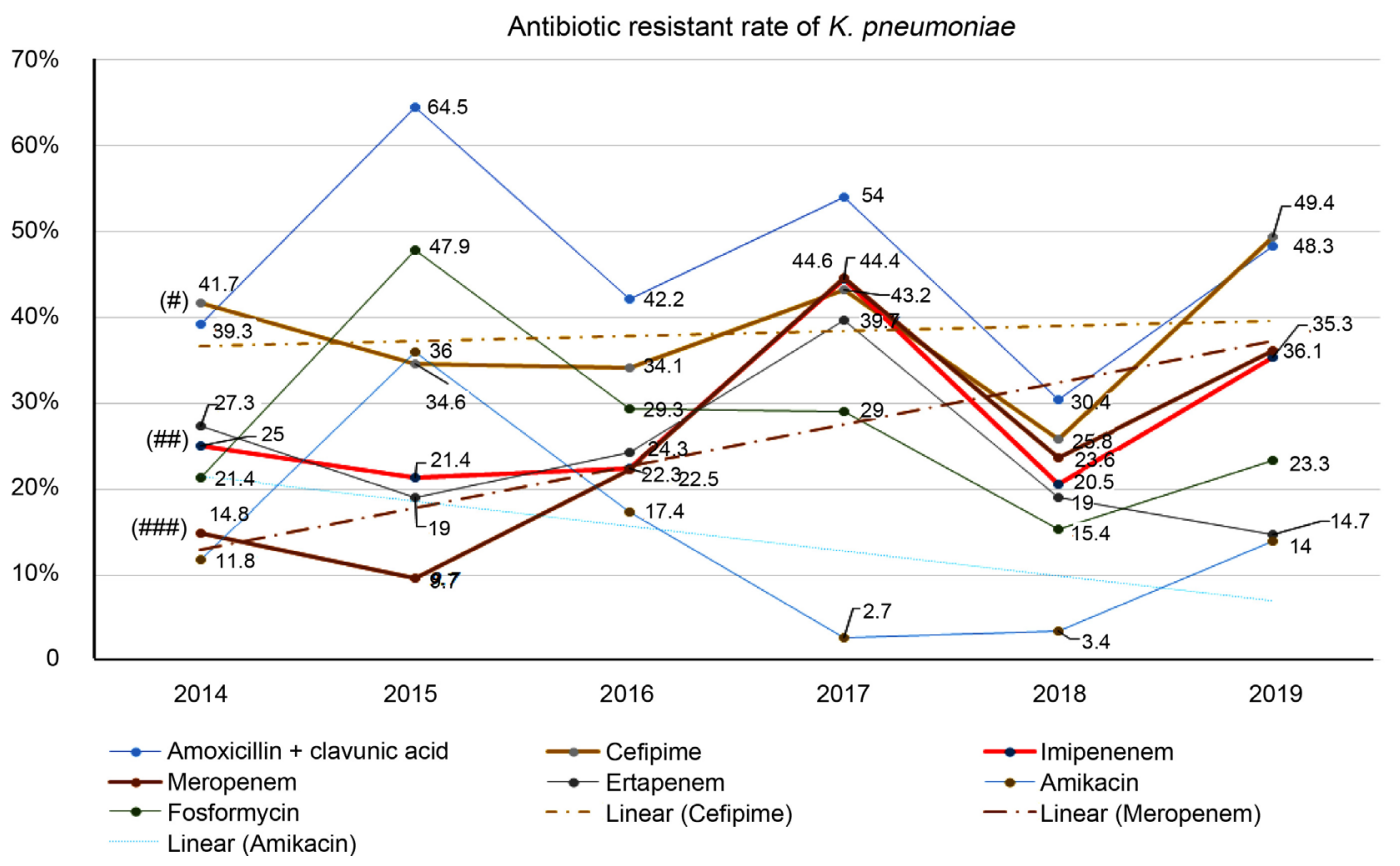

Figure 6. The resistant level of $K$. pneumonia over time. ${ }^{*} \mathrm{p}<0.05$, a statistically significant result in Cefipime resistance during 2014-2019. ${ }^{\# \#} \mathrm{p}<0.05$, a statistically significant result in Imipenenem resistance during 2014-2019. ${ }^{\# \#} \mathrm{p}<0.05$, a statistically significant result in Meropenem resistance during 2014-2019. 


\section{Discussion}

In the short time the antibiotic was discovered and put into treating infections, the bacteria was able to resist them. It was found that the more antibiotics used, the faster they became resistant. In the race between finding a new antibiotic and the speed of increasing antibiotic resistance of bacteria, it seemed that bacteria always took the initiative. The natural selection pressure and the struggle for survival among bacteria have helped them an antibiotic resistant ability. Moreover, the combination of antibiotics in treatment led to multidrug resistance of bacteria [12].

During the study, we found that the percentage of more than 3-antibiotic group resistant bacteria was higher than the rate of less than 3-antibiotic group resistant bacteria in all four bacterial species. In partially, the rate of multi-resistant bacteria has increased over time. It led to a difficulty in selecting antibiotics for infection treatment in our hospital. Comparing with Japanese data in the 2018 annual monitoring report, the proportion of antibiotic resistant bacteria in 103 military hospital of Vietnam was much higher than the Japanese hospital (Table 1) [13].

The research and classify MDR, XDR and PDR strains were followed to the standards of the European Center for Disease Control and Prevention (ECDC) [12]. In this study, it was really worried that in all four common gram-negative bacilli, the rates of multi-drug resistant bacteria were more than $50 \%$ and this percentage had been increasing during the study period. For example, the multi-drug resistant rate of $A$. baumannii was $79.57 \%$ in 2017 and this figure was $86.8 \%$ in 2019. Moreover, MDR bacteria (non XDR or pPDR - resisted to more

Table 1. Comparison of antibiotic resistant bacteria strains between 103 military hospital and Japanese.

\begin{tabular}{|c|c|c|c|}
\hline & $\begin{array}{l}\text { The } 103 \text { military hospital } \\
\qquad(\mathrm{n}=2209)\end{array}$ & $\begin{array}{c}\text { Japan } \\
(\mathrm{n}=2,891,652)\end{array}$ & $p$ \\
\hline $\begin{array}{l}\text { Multidrug-resistant } \\
P \text {. aeruginosa }\end{array}$ & $\begin{array}{c}242 \\
(11.0 \%)\end{array}$ & $\begin{array}{c}1082 \\
(0.04 \%)\end{array}$ & $<0.05$ \\
\hline $\begin{array}{l}\text { Multidrug-resistant } \\
\text { Acinetobacter spp. }\end{array}$ & $\begin{array}{c}257 \\
(11.6 \%)\end{array}$ & $\begin{array}{c}99 \\
(0.00 \%)\end{array}$ & $<0.05$ \\
\hline $\begin{array}{l}\text { Carbapenem-resistant } \\
\text { Enterobacteriaceae }\end{array}$ & $\begin{array}{c}189 \\
(8.6 \%)\end{array}$ & $\begin{array}{c}9304 \\
(0.32 \%)\end{array}$ & $<0.05$ \\
\hline $\begin{array}{l}\text { Carbapenem-resistant } \\
P \text {. aeruginosa }\end{array}$ & $\begin{array}{c}199 \\
(9.0 \%)\end{array}$ & $\begin{array}{c}21202 \\
(0.73 \%)\end{array}$ & $<0.05$ \\
\hline $\begin{array}{l}3^{\text {rd }} \text { Generation } \\
\text { Cephalosporin-resistant } E \text {. coli }\end{array}$ & $\begin{array}{c}342 \\
(15.5 \%)\end{array}$ & $\begin{array}{l}92653 \\
(3.2 \%)\end{array}$ & $<0.05$ \\
\hline $\begin{array}{l}3^{\text {rd }} \text { Generation } \\
\text { Cephalosporin-resistant } K \text {. } \\
\text { pneumonia }\end{array}$ & $\begin{array}{c}136 \\
(6.2 \%)\end{array}$ & $\begin{array}{c}14858 \\
(0.51 \%)\end{array}$ & $<0.05$ \\
\hline $\begin{array}{l}\text { Fluoroquinolone-resistant } \\
\text { E. coli }\end{array}$ & $\begin{array}{c}321 \\
(14.5 \%)\end{array}$ & $\begin{array}{l}133170 \\
(4.61 \%)\end{array}$ & $<0.05$ \\
\hline
\end{tabular}


than 3 antibiotic groups) have been replaced by XDR (only sensitive with one or two antibiotic groups) or possible PDR (may be resist all antibiotics) strains. In 2014, among the multidrug resistant $P$. aeruginosa strains, the XDR strain accounted for $67.86 \%$, in 2019 this number increased to $90.32 \%$ (Figure 2). A multicenter study in Mexico that analyzed and assessed microbiological data from 47 units over 6 months showed that K. pneumoniae MDR (22.6\%), E. coli (19.4\%), Enterobacter spp (11.9\%) and P. aeruginosa (8.8\%). A. baumannii had the highest rate of XDR (8.8\%) and no PDR strain has been detected [14] [15]. The announcement of an emergency resuscitation department in Tertiary India about the percentage of multidrug resistant $P$. aeruginosa strains, the MDR strains was $50 \%$, XDR strains was $2.3 \%$ and no PDR strains found [15]. A survey of resistant bacteria trends in Mexico from 2005 to 2012 was also showed that $A$. baumannii increased MDR rate from $25.6 \%$ to $49.7 \%$ [6]. Although PDR strain had not found in the time of the study, the antibiotic resistant level of bacteria in this study was much higher than several studies in the world. This problem has warned a risk of transmission of multidrug-resistant strains, so we have to control closely hospital infections.

It can be said that $A$. baumannii was one of the most mentioned bacteria in the last 15 - 20 years, because it was the most serious nosocomial infection agent and resisted many different kinds of antibiotics. Therefore, WHO has been prioritized in research new antibiotics [2]. The results showed that the antibiotic resistant rates of $A$. baumannii were large with all most antibiotic groups such as cephalosporins, aminoglycosides, and fluoroquinolones. Even with carbapenem, which is a strong antibiotic, $A$. baumannii was not only resistant more than $50 \%$ but also increased over time. However, A. baumannii resisted amikacin and co-trimoxazole with low rates and decreased slowly every year. It is a good sign in selection of antibiotics for treatment $A$. baumanni (Figure 3). Other studies were shown the similar results in study $A$. baumannii from different areas [4] [5] [14] [16] [17] [18]. However, in a report from 2013 to 2017 of Malaysia were showed a difference. For example, $A$. baumannii was lower resistant imipenem, meropenem, and ceftazidime than our results with ranging from $55 \%-62 \%$, and it also resisted amikacin with the higher rates from $42 \%$ to $50 \%$ [19]. In another research from Mexico between 2005 and 2012 showed that the resistant percentage of $A$. baumannii to meropenem was from $17.7 \%$ to $33.0 \%$, was not as high as the figure in this research [6]. In significant, in different time and regions the antibiotic resistant level of $A$. baumannii was different. Therefore, the monitoring of drug resistance should be conducted regularly and continuously.

Similar to A. baumannii, $P$. aeruginosa had a high level of resistance and increasing tendency with many antibiotics over time. This is a challenge in treating these strains in the near future. Here results were quite the same as the results from other studies [4] [20]. According to this research, E. coli were generally resistant rate lower than that of $A$. baumannii and $P$. aeruginosa. The rate of resistance increased over time such as cefotaxim, ciprofloxacin, and co-trimoxazole. 
However, other antibiotics as amoxicillin/clavunanic acid, cefepime, fosformycin, nitrofurantoin, and amikacin decreased slowly the rate of resistance. Fortunately, the amikacin, fosformycin and carbapenem antibiotics are also good choices for clinicians because of high sensitivity. Nguyen Vinh Nghi in Ninh Thuan General Hospital of Vietnam was reported E. coli was resistant cephalosporin with the rate of $48.2 \%$ to $75 \%$, and quinolone $72.7 \%$ and $73.2 \%$ higher than our research results, because these antibiotics have been used popularly in recent years. However, the resistant rates of $E$. coli with carbapenem and aminoglycoside were $0.7 \%$ and $1.4 \%$, lower than this study [21]. The reason for this difference was probably these two antibiotics from reserve group. Provincial hospitals rarely used them for treatment meanwhile they were applied in most of the central hospitals. In South Korea, the 2017 surveillance report showed that the levels of antibiotic resistance of $E$. coli isolated from blood samples were $65.3 \%, 32.4 \%, 11.8 \%$ and $20.3 \%$ to ampicillin, cefotaxim, ceftazidim and cefepime respectively, whereas, the rates of carbapenem and amikacin were very low, accounting for $0.2 \%$ and $0.8 \%$ [22]. In other countries such as Japan and Malaysia, the rates of carbapenem and amikacin resistance of $E$. coli remained lower than $2 \%$ over the years and lower than $30 \%$ with cephalosporin [13] [19]. In some countries such as Russia, Serbia, Switzerland, Montenegro, and Ukraine, the rates of carbapenem resistance of $E$. coli isolated from blood and cerebrospinal fluid samples were lower than 2\% [23]. E. coli is an agent of the intestinal microflora and it can cause opportunistic diseases, especially nosocomial infections. Here, the drug resistant levels of $E$. coli were bigger than other studies from different countries, probably Vietnam is a developing country, and environmental sanitation is not really good.

Like E. coli, A. baumannii, and $P$. aeruginosa, $K$. pneumoniae also plays an important role in nosocomial infections. According to this research, $K$. pneumoniae had high levels of resistance with many different antibiotics and higher resistant level than other studies [19] [20] [21] [22] [24] [25]. The carbapenem resistant rate increased significantly from $10 \%$ to $45 \%$. Fortunately, K. pneumoniae still tended to significantly reduce the resistance with some antibiotics like ertapenem, fosfomycin, amikacin, and amoxicillin/calvulanic acid. It can be said that selecting antibiotics for treatment of $A$. baumannii and $P$. aeruginosa strains are more difficult than choosing drug in treatment for E. coli and K. pneumoniae strains.

\section{Conclusions}

Although the study was conducted in only a hospital, it is a military central hospital in Vietnam, where treated for a lot of serious patients with diverse diseases from different locals in the North of Vietnam. Thus, it can reflect the disease situation in a big area. The regular update on the level of antibiotic resistance of Gram-negative bacteria in 103 Military Hospital is always necessary. It provides useful information for clinical physicians in selecting antibiotics for treatment, 
contributes in improving the quality of treatment and limiting the spread of antibiotic-resistant bacteria strains. In summary, our results indicated a trend of increasing resistance over time and a high resistant rate with many antibiotics of Gram-negative strains at a military hospital in Vietnam. In general, The XDR or pPDR strains had accounted a great proportion. It was a major challenge in the selection of antibiotics for treatment. However, results also showed that each bacterium was susceptible with some antibiotics. Depending on these results, clinicians have the best decision in choosing antibiotics for treatment as well as establishing appropriate strategies in using antibiotic. It is essential to regularly monitor of bacterial resistant levels, and should pay attention to more in-depth studies related to drug resistance such as resistance mechanisms, transmission routes of resistance strains and intensify control of nosocomial infections to limit the spread of multi-resistant strains.

\section{Acknowledgements}

This research is grateful to the Medical Microbiology Department, Vietnam Military Medical University for assistance in collecting samples.

\section{Conflicts of Interest}

The authors declare no conflicts of interest regarding the publication of this paper.

\section{References}

[1] Daxboeck, F., Budic, T., Assadian, O., Reich, M. and Koller, W. (2006) Economic Burden Associated with Multi-Resistant Gram-Negative Organisms Compared with That for Methicillin-Resistant Staphylococcus aureus in a University Teaching Hospital. Journal of Hospital Infection, 62, 214-218. https://doi.org/10.1016/j.jhin.2005.07.009

[2] WHO (2017) Global Priority List of Antibiotic Resistant Bacteria to Guide Research, Discovery, and Development of Antibiotics. Geneva.

[3] Zhen, X., Lundborg, C.S., Sun, X., Hu, X. and Dong, H. (2019) Economic Burden of Antibiotic Resistance in ESKAPE Organisms: A Systematic Review. Antimicrobial Resistance and Infection Control, 8, 137.

https://doi.org/10.1186/s13756-019-0590-7

[4] Nhung, P.H. (2018) Analysis of Antibiotic Resistance Situation of Klebsiella pneumoniae, Pseudomonas aeruginosa and Acinetobacter baumannii Isolated at Intensive Care Unit and Respiratory Center of Bach Mai Hospital, 2012-2016. Clinical Medicine Journal, 101, 43-50.

[5] Phu, V.D., Wertheim, H.F., Larsson, M., Nadjm, B., Dinh, Q.D., Nilsson, L.E., Rydell, U., Le, T.T., Trinh, S.H., Pham, H.M., Tran, C.T., Doan, H.T., Tran, N.T., Le, N.D., Huynh, N.V., Tran, T.P., Tran, B.D., Nguyen, S.T., Pham, T.T., Dang, T.Q., Nguyen, C.V., Lam, Y.M., Thwaites, G., Van Nguyen, K. and Hanberger, H. (2016) Burden of Hospital Acquired Infections and Antimicrobial Use in Vietnamese Adult Intensive Care Units. PLoS ONE, 11, e 0147544. https://doi.org/10.1371/journal.pone.0147544

[6] Morfin, O.R., Noriega, E.R. and Dowzicky, M.J. (2015) Antimicrobial Susceptibility 
Trends among Gram-Positive and -Negative Clinical Isolates Collected between 2005 and 2012 in Mexico: Results from the Tigecycline Evaluation and Surveillance Trial. Annals of Clinical Microbiology and Antimicrobials, 14, 53. https://doi.org/10.1186/s12941-015-0116-y

[7] Leber, A.L. (2016) Clinical Microbiology Procedures Handbook.

[8] MOH (2017) Practical Guidelines for Clinical Microbiology Laboratory Techniques. Medical Publishing, Hanoi.

[9] Nimer, N.A., Al-Saa'da, R.J. and Abuelaish, O. (2016) Accuracy of the VITEK(R) 2 System for a Rapid and Direct Identification and Susceptibility Testing of Gramnegative Rods and Gram-Positive Cocci in Blood Samples. Eastern Mediterranean Health Journal, 22, 193-200. https://doi.org/10.26719/2016.22.3.193

[10] Nachnani, S., Scuteri, A., Newman, M.G., Avanessian, A.B. and Lomeli, S.L. (1992) E-Test: A New Technique for Antimicrobial Susceptibility Testing for Periodontal Microorganisms. Journal of Periodontology, 63, 576-583.

https://doi.org/10.1902/jop.1992.63.7.576

[11] CLSI (2018) Performance Standards for Antimicrobial Susceptibility Testing. Clinical and Laboratory Standards Institute.

[12] Magiorakos, A.P., Srinivasan, A., Carey, R.B., Carmeli, Y., Falagas, M.E., Giske, C.G., Harbarth, S., Hindler, J.F., Kahlmeter, G., Olsson, L.B., Paterson, D.L., Rice, L.B., Stelling, J., Struelens, M.J., Vatopoulos, A., Weber, J.T. and Monnet, D.L. (2012) Multidrug-Resistant, Extensively Drug-Resistant and Pandrug-Resistant Bacteria: An International Expert Proposal for Interim Standard Definitions for Acquired Resistance. Clinical Microbiology and Infection, 18, 268-281. https://doi.org/10.1111/j.1469-0691.2011.03570.x

[13] Japan, N. I. o. I. D. (2019) Annual Open Report 2018. Japan Nosocomial Infections Surveillance (JANIS).

[14] Gonzalez, E.G., Otero, R.M., Olazaran, S.M., Ibarias, P.B., Trevino, S.F., Noriega, E.R., de Leon, A.P., Francia, D.S., Cendejas, R.F., Escalante, S.A., Acosta, C.V., Larios, F.R., Quintanilla, L.J., Anicacio, J.Y.M., Miranda, R.M., Cantu, H.L.O., Choel, A.G., Sanchez, J.L.J., Benıtez, L.K.A., Guzman, J.M.F., Lopez, C.D.P., May, C.A.C., Jaimes, A.M., Narvaez, E.G.V., Zuno, J.R., Garay, R.R., Espinoza, A.G., Ramirez, A.M., Mora, J.P., Rojas, R.E.C., Ovilla, I.L., Colin, V.A.M., Magallon, J.M.B., De laPena, C.T.M., Burciaga, E.A., Ramirez, M.C., Garcia, A.A.R., Ayala, M.T., Rodrıguez, S.S., Vega, B.A.P., Rodrıguez, A.N., Velazquez, G.E.J., Umana, C.M.C., Ramırez, J.P.M., Oviedo, J.C., Mendez, M.I.M., Romero, D.R., Mejia, A.A., Canul, D.I.C., Orozco, G.A., Sanchez, J.S. and Ortiz, A.C. (2019) A Snapshot of Antimicrobial Resistance in Mexico. Results from 47 Centers from 20 States during a Six-Month Period. PLoS ONE, 14, e0209865.

https://doi.org/10.1371/journal.pone.0209865

[15] Gill, J.S., Arora, S., Khanna, S.P. and Kumar, K.H. (2016) Prevalence of Multidrug-Resistant, Extensively Drug-Resistant, and Pandrug-Resistant Pseudomonas aeruginosa from a Tertiary Level Intensive Care Unit. Journal of Global Infectious Diseases, 8, 155-159. https://doi.org/10.4103/0974-777X.192962

[16] An, V.D. (2018) The Situation of Pneumonia Related to Mechanical Ventilation at the Intensive Care Unit of Military Hospital 175. Ho Chi Minh Medical Journal, 22, 55-61.

[17] Bao, N.T., Nhi, L.T.A.P., Nga, V.T.T., Linh, D.T.D. and Binh, D.T. (2018) Gram-Negative Bacilli Do Not Ferment Sugars in Bacterial Infections and Antibiotic Resistance. Medicine City Ho Chi Minh, 2, 22. 
[18] Ngo, T.V., Thao, P.T.N. and Nga, T.T.T. (2017) Survey on Resistance Characteristics of Pseudomonas aeruginosa and Acinetobacter baumannii Causing Pneumonia in Hospital. Ho Chi Minh City Medical Association, Ho Chi Minh City, 61-64.

[19] Unit IaB (2017). Nation Antibiotic Resistance Surveillance Report 2017. Infectious Diseases Research Centre Institute for Medical Research: Kuala Lumpur, Malaysia.

[20] Lan, N.N., Nga, C.M. and Kieu, N.T.T. (2018) Resistance of Pathogenic Bacteria Is Often Found in Lower Respiratory Tract Specimens at the Ho Chi Minh University Hospital City for a Year (May 1, 2016-April 30, 2017). Ho Chi Minh Medical Journal, 22, 382-389.

[21] Nghi, N.V. (2017) The Situation of Antibiotic Resistance of Common Bacterial Strains in Ninh Thuan Hospital since 2017. Time of Medicine, December, 40-46.

[22] Liu, C., Yoon, E.J., Kim, D., Shin, J.H., Shin, J.H., Shin, K.S., Kim, Y.A., Uh, Y., Kim, H.S., Kim, Y.R. and Jeong, S.H. (2019) Antimicrobial Resistance in South Korea: A Report from the Korean Global Antimicrobial Resistance Surveillance System (Kor-GLASS) for 2017. Journal of Infection and Chemotherapy, 25, 845-859. https://doi.org/10.1016/j.jiac.2019.06.010

[23] DANMAP (2018) Use of Antimicrobial Agents and Occurrence of Antimicrobial Resistance in Bacteria from Food Animals, Food and Humans in Denmark. National Food Institute, Technical University of Denmark.

[24] Huyen, M.N.T., Nguyen, D.D. and Lan, N.H. (2018) The Common Bacteria and Their Antibiotic Resistance at Pham Ngoc Thach Hospital from 11/2016 to 11/2017. Ho Chi Minh Medical Journal, 22, 196-200.

[25] Wang, C., Yuan, Z., Huang, W., Yan, L., Tang, J. and Liu, C.W. (2019) Epidemiologic Analysis and Control Strategy of Klebsiella pneumoniae Infection in Intensive Care Units in a Teaching Hospital of People's Republic of China. Infection and Drug Resistance, 12, 391-398. https://doi.org/10.2147/IDR.S189154 